\title{
Magnesium Acetate Tetrahydrate
}

National Cancer Institute

\section{Source}

National Cancer Institute. Magnesium Acetate Tetrahydrate. NCI Thesaurus. Code C48001.

The hydrated acetate salt form of magnesium. Magnesium is a divalent cation essential for a number of biochemical processes involved in nerve signaling, bone mineralization and muscle contractions. About 350 enzymes involved in glycolysis and the Krebs cycle, formation of cyclic-AMP and ATP, cellular signal transduction and protein and nucleic acid synthesis are dependent on magnesium. 